\title{
Immigration et morphologie des parcours scolaires dans l'enseignement supérieur au Canada : le cas de la province de Québec
}

Morphology of Immigrant Youth Pathways in Canadian Higher Education: The Case of the Province of Quebec Inmigración y morfología de las trayectorias académicas en la educación superior en Canadá: el caso de la provincia de Quebec

Pierre-Canisius Kamanzi, Marie-Odile Magnan, Annie Pilote et Pierre Doray

\section{OpenEdition}

\section{Journals}

Édition électronique

URL : https://journals.openedition.org/remi/11280

DOI : $10.4000 /$ remi. 11280

ISSN : $1777-5418$

Traduction(s) :

Immigrant Youth in Canadian Postsecondary Education: Pathway Morphologies in the Province of Quebec - URL : https://journals.openedition.org/remi/12100 [en]

Éditeur

Université de Poitiers

Édition imprimée

Date de publication : 1 octobre 2018

Pagination : 253-277

ISBN : 979-10-90426-62-7

ISSN : 0765-0752

Référence électronique

Pierre-Canisius Kamanzi, Marie-Odile Magnan, Annie Pilote et Pierre Doray, «Immigration et morphologie des parcours scolaires dans l'enseignement supérieur au Canada : le cas de la province de Québec », Revue européenne des migrations internationales [En ligne], vol. 34 - n² et 3 | 2018, mis en ligne le 01 janvier 2021, consulté le 14 avril 2022. URL : http://journals.openedition.org/remi/11280 ; DOI : https://doi.org/10.4000/remi. 11280 


\section{Immigration et morphologie des parcours scolaires dans l'enseignement supérieur au Canada : le cas de la province de Québec}

\section{Pierre Canisius Kamanzi ${ }^{1}$, Marie-Odile Magnan², Annie Pilote ${ }^{3}$ et Pierre Doray ${ }^{4}$}

Au cours des dernières décennies, les pays industrialisés ont été la destination de vagues importantes d'immigration (OCDE, 2017 ; OECD, 2015). Selon le dernier rapport de l'OCDE (2017), environ 124 millions de personnes résidant dans ses pays membres sont nées à l'étranger, soit à peu près $13 \%$ de la population totale contre $9,5 \%$ en 2000 . Le même rapport indique qu'après les États-Unis, l'Allemagne et le Royaume-Uni, le Canada est le pays ayant reçu le plus d'immigrants durant la période 2004-2015, soit en moyenne 250000 personnes chaque année. En 2015, par exemple, le pays a enregistré 272000 nouveaux résidents permanents. De tous les pays, c'est au Canada et en Australie que l'immigration constitue la part la plus importante de la population (Ferrer et al., 2014). En 2016, I'effectif des immigrants canadiens ${ }^{5}$ était de 7540830 , soit $22 \%$ de la population totale (35151728) (Statistique Canada, 2017b). S'ils étaient disséminés dans toutes les provinces et territoires du pays, la majorité des immigrants résidaient cependant dans trois provinces : 3852145 (51\%) en Ontario, 1292675 (17\%) en Colombie-Britannique et 1091310 (15\%) au Québec. Autrement dit, à elles seules, ces trois provinces cumulaient 6236130 , soit $83 \%$

\footnotetext{
1 Professeur agrégé, Département d'administration et fondements de l'éducation, Université de Montréal, Pavillon Marie-Victorin, bureau A-511, C.P. 6128, succursale Centre-ville, Montréal, Canada H3C 3J7; pierre.canisius.kamanzi@umontreal.ca

2 Professeure agrégée, Département d'administration et fondements de l'éducation, Université de Montréal, Pavillon Marie-Victorin, bureau A-523, C.P. 6128, succursale Centre-ville, Montréal, Canada H3C 3J7 ; marie-odile.magnan@umontreal.ca

3 Professeure titulaire, Faculté des sciences de l'éducation, Pavillon des sciences de I'éducation, 2320 rue des Bibliothèque, Université Laval, Québec, Canada G1V 0A6 ; annie.pilote@fse.ulaval.ca

4 Professeur titulaire, Département de sociologie, Université du Québec à Montréal (UQAM), CP 8888, succursale Centre-ville, Québec, Canada H3C3P8 ; doray.pierre@uqam.ca

5 Selon Statistique Canada (2017b), le terme "immigrants " réfère à toute personne qui est, ou a été immigrant reçu ou résident permanent. II s'agit de toute personne à qui les autorités de l'immigration ont accordé le droit de résider au Canada en permanence. Les immigrants qui ont obtenu la citoyenneté canadienne par naturalisation sont compris dans cette catégorie.
} 
de la population immigrée. Par ailleurs, à l'intérieur de ces trois provinces, presque un tiers $(27,4 \%)$ de ces immigrants résidait dans les trois grandes villes du pays : 1266005 (17\%) à Toronto (Ontario), 570940 (7\%) à Montréal (Québec) et 262765 (3,4 \%) à Vancouver (Colombie-Britannique).

Les immigrants canadiens se répartissent en trois catégories principales : les immigrants économiques (les travailleurs qualifiés, les investisseurs), les réfugiés et ceux qui arrivent par la voie de la réunification familiale (Statistique Canada, 2017b). Par rapport aux autres membres de I'OCDE, le Canada considère davantage l'immigration comme un moyen de combler les besoins de maind'œuvre qualifiée, et ce, depuis plusieurs décennies (Ferrer et al., 2014). La catégorie "immigrants économiques" représente ainsi à peu près $60 \%$ de I'ensemble des immigrants (Martel et D'Aoust, 2016). Bien que cette catégorie comprenne également les investisseurs sélectionnés en fonction du volume des fonds qu'ils s'apprêtent à investir, la plupart sont de futurs travailleurs et sont sélectionnés sur la base de différents critères de compétences professionnelles, dont les principaux sont le niveau de scolarité et de qualification, la connaissance de l'anglais ou du français, l'âge, l'expérience professionnelle. Cela étant, quelle que soit la catégorie à laquelle ils appartiennent, les nouveaux arrivants sont perçus comme un capital humain potentiel qu'il faut rentabiliser à court ou long terme (Ferrer et al., 2014). Selon les économistes, le plus tôt ils sont intégrés dans les structures de la société, le mieux ils sont à même de mettre jour leur capital humain et participer à l'activité économique (Djajic, 2003) et telle est la perspective des politiques d'immigration canadiennes (Ferrer et al., 2014).

L'intensification des mouvements migratoires dans les pays industrialisés se caractérise non seulement par l'accroissement des effectifs des immigrants, mais également par la diversité de leurs origines. Si par le passé, l'immigration s'opérait généralement entre les pays eux-mêmes, le plus souvent entre des États voisins et frontaliers, les flux migratoires se sont diversifiés et élargis aux pays du Sud depuis la fin du siècle dernier (Zlotnik, 1991 et 1995), particulièrement dans les pays asiatiques (OCDE, 2017). Pour le Canada, environ $60 \%$ d'entre eux viennent des pays de l'Asie, $17 \%$ de l'Afrique et $12 \%$ de l'Europe (Martel et D'Aoust, 2016). Toutefois, la situation varie entre les provinces : par rapport au reste du Canada, le Québec accueille davantage de nouveaux arrivants de I'Afrique, et moins de l'Asie. Les ressortissants de l'Afrique du Nord occupent le premier rang, ce qui témoigne entre autres de l'objectif du gouvernement québécois d'accroître la proportion des immigrants francophones (MIDI, 2015). Une telle différence s'explique en grande partie par les dispositions de la constitution canadienne qui prévoit le partage des compétences en matière d'immigration entre les niveaux fédéral et provincial à partir des années 1960 et plus encore des années 1970. Bien que la législation fédérale soit prépondérante, le Québec détient d'importantes compétences pour sélectionner, accueillir et intégrer les immigrants en fonction de ses propres politiques de démographie (MIDI, 2015).

Cette hétérogénéité croissante des immigrants au plan ethnoculturel dans les pays développés n'est pas sans poser d'importants défis d'intégration dans les pays d'accueil (Koopmans, 2010). Ainsi, malgré l'égalité formelle des chances d'accès à l'école, plusieurs études menées dans les pays développés témoignent des inégalités persistantes entre les élèves issus de familles immigrées et leurs 
pairs dont les parents sont natifs du pays d'accueil. Si une partie des jeunes d'origine immigrante parvient à terminer la scolarité secondaire en raison de son caractère obligatoire, la majorité est contrainte de réduire ses ambitions à cause de la sélection scolaire (Baum et Flores, 2011; Griga et Hadjar, 2013). La sélection et l'orientation scolaires précoces sont telles que seule une minorité accède aux filières ou aux programmes donnant accès à l'enseignement supérieur, tandis que la majorité est reléguée dans des filières professionnelles conduisant au marché du travail. Ces personnes sont le plus souvent contraintes de mettre fin à leur scolarité (Crul et al., 2012).

La situation varie cependant entre pays et, à cet égard, le Canada fait partie des exceptions (Jenkins et al., 2008). Comme nous le verrons dans la section suivante, nombre de travaux soulignent qu'il est l'un des rares pays où les élèves d'origine immigrantes ont des performances scolaires comparables à celles de leurs pairs non immigrants (OECD, 2015; Schnepf, 2007). Qui plus est, les jeunes issus de l'immigration accèdent aux études supérieures dans une proportion plus élevée que leurs pairs dont les parents sont nés au Canada (Finnie et Mueller, 2010; Picot, 2012; Thiessen, 2009). Toutefois, des inégalités persistent entre les communautés immigrées, comme en témoignent d'autres travaux (Abada et Tenkorang, 2009; Kamanzi et al., 2016; Kamanzi et Murdoch, 2011 ; Thiessen, 2009). Certaines communautés ethniques d'origine immigrantes dites racisées, c'est-à-dire, cibles de racisme, sont encore confrontées à des formes de ségrégations mitigées (Henry et Tator, 2009 et 2010).

Ces constats observés dans l'ensemble du Canada ont été mis en évidence au Québec (Mc Andrew et al., 2015; Mc Andrew et al., 2008). Les auteurs montrent que les jeunes d'origine immigrante ont, de manière générale, des parcours scolaires comparables à ceux de leurs pairs dont les parents sont non immigrants, mais que des écarts importants entre les communautés persistent. D'un côté, les jeunes dont les parents viennent des pays européens, asiatiques ou arabes ont des parcours scolaires comparables, voire exceptionnels, comparativement à ceux de leurs pairs dont les parents sont natifs du Canada. D'un autre côté, ceux dont les parents viennent de l'Afrique subsaharienne, des Caraïbes et de l'Amérique latine tendent à avoir des parcours difficiles au secondaire et ont des taux de réussite plus bas que la moyenne provinciale. Par ailleurs, ils sont moins enclins à fréquenter les écoles privées sélectives que les jeunes originaires d'autres régions (Mc Andrew et al., 2011). En particulier, les jeunes issus des Caraïbes et de l'Afrique subsaharienne sont également plus susceptibles d'avoir vécu des situations d'exclusion et de discrimination pendant leurs études primaires et secondaires (Lafortune, 2012).

Dès lors que les performances scolaires varient selon l'origine des parents des élèves, cette étude pose l'hypothèse selon laquelle ces disparités de réussite à l'enseignement de base se traduisent par l'existence de différences de parcours aux études supérieures. Plus précisément, tout indique que les cheminements dans l'enseignement supérieur varient selon l'origine géographique des parents d'élèves. Avant de valider cette hypothèse, nous proposons un bref aperçu sur le contexte de la scolarisation des immigrants au Canada, un cadre d'analyse reposant sur le concept de parcours scolaire et une description sommaire de la structure du système scolaire du Québec. 


\section{Les jeunes issus de l'immigration et I'offre de parcours scolaires au Canada}

Alors que l'intégration sociale des immigrants est en grande partie évaluée en fonction de l'accès à des emplois de qualité, qui lui-même repose essentiellement sur le niveau et la qualité de leur formation (Ichou, 2014), les études à l'échelle internationale soulignent les ségrégations dont ils font l'objet dans les systèmes scolaires des pays d'accueil (Levels et al., 2008; Söhn et Özcan, 2006), voire de marginalisation directe dans certains contextes (Felouzis et al., 2005; Cutler et al., 2007). De génération en génération, ces ségrégations perpétuent des inégalités scolaires et sociales entre les jeunes issus des communautés immigrantes et leurs pairs issus des familles natives de la société d'accueil. Les premiers ont des résultats scolaires plus faibles dans l'enseignement de base (primaire et secondaire), comme en témoignent plusieurs études comparatives à partir des résultats des tests internationaux (Dronkers et van der Velden, 2013; Dustmann et al., 2011; Hochschild et Croopper, 2010; OCDE, 2006; Schnepf, 2007). Par la suite, les chances d'accéder aux études supérieures et d'y obtenir un diplôme sont réduites pour les jeunes d'origine immigrante. Toutefois, la situation varie considérablement selon le contexte sociétal, comme le révèlent les études précitées. Si les écarts sont nettement plus élevés dans les pays comme la Belgique, l'Allemagne, la Suisse et la France, pour ne citer que quelques-uns, ils semblent relativement modérés dans les pays anglo-saxons (États-Unis, Canada, Australie et Nouvelle-Zélande) (Buchmann et Parrado, 2006). À cet égard, l'Australie et le Canada constituent des exceptions : les élèves d'origine immigrante ont des performances scolaires comparables à celles de leurs pairs non immigrants.

Dans le cas du Canada, une première explication a été proposée pour comprendre cette situation exceptionnelle : les dispositions mêmes de ses politiques d'immigration qui sélectionnent les immigrants sur la base de leur capital scolaire (Boyd, 2002) et qui privilégient l'intégration et le multiculturalisme plutôt que I'assimilation et le monoculturalisme (Ghosh, 2004; Harles, 1997 et 2004). Cet engagement politique, enchâssé dans la Loi sur le multiculturalisme canadien, se concrétise par une culture de tolérance (Bloemraad, 2012; Li, 2003).

Contrairement à certains pays semblables, les politiques canadiennes d'immigration sont axées sur le multiculturalisme, c'est-à-dire sur le respect et la valorisation des différences culturelles. Sur le plan local, la réussite des immigrants serait attribuable au principe et aux mesures d'équité. Qu'ils soient nés au Canada ou à l'étranger, tous les élèves canadiens d'origine immigrante fréquentent inconditionnellement les mêmes établissements que les non-immigrants et bénéficient de la même formation. En outre, cette égalité d'accès est renforcée par l'offre de ressources et services supplémentaires visant à soutenir l'intégration des nouveaux arrivants ou ceux ayant des difficultés particulières (Shakya et al., 2010; Smich et al., 2005). Parmi ces mesures, on peut citer les classes d'accueil, le soutien linguistique en classe régulière et les cours gratuits de français ou d'anglais pour les nouveaux arrivants - les enfants comme les parents - selon la province ou le territoire de résidence.

Or, force est de constater que cette volonté politique est loin d'être évidente dans plusieurs pays. En effet, certains d'entre eux tolèrent, voire légitiment à des 
degrés variés la culture et les pratiques de marginalisation des élèves immigrants (Buchmann et Parrado, 2006). Dans plusieurs pays européens, les élèves d'origine immigrante sont concentrés et marginalisés dans des établissements de quartiers défavorisés où l'enseignement est de moindre qualité (Schnell et Azzolini, 2015), comme en témoignent, par exemple, les études de Auernheimer (2006) en Allemagne, de Schneeweiss (2013) en Autriche, ainsi que de Schnell et Azzolini (2015) dans les pays méditerranéens (Italie, Grèce, Portugal et Espagne).

La deuxième explication qui complète la première réfère aux caractéristiques des immigrants eux-mêmes et aux critères de leur sélection. Comme souligné dans l'introduction, la majorité des immigrants canadiens font partie de la catégorie économique et sont sélectionnés sur la base de leur niveau de scolarité et de leurs compétences professionnelles. Ils sont en moyenne plus scolarisés que le reste de la population (King et Newbold, 2007). Les études (Lowell, 2005; Narada et Rieger, 2010) montrent que le Canada, I'Australie et le Royaume-Uni sont les trois pays qui accordent davantage d'importance à ces critères au moment de la sélection. Dès lors, les performances scolaires des élèves immigrants seraient, en grande partie, attribuables à l'influence de la scolarité de leurs parents. Celle-ci constitue une source de différentes formes de capitaux (social, culturel) mobilisés par les familles et les élèves dans leur scolarisation (Boyd, 2002; Hochschild et Cropper, 2010; Rothon et al., 2009). Pour plusieurs familles immigrantes, la scolarisation de leurs enfants constitue presque le seul moyen d'envisager une intégration sociale dans le pays de destination et même une mobilité sociale qui leur permettra de recouvrer leur statut socioéconomique antérieur à l'immigration (Ichou, 2014). Même si les politiques canadiennes favorisent l'intégration professionnelle des immigrants, ces derniers ne sont pas à l'abri des ségrégations et ont souvent besoin d'être plus scolarisés que leurs concitoyens pour accéder à des emplois équivalents (Reitz, 2001). Dès lors que l'accès à l'enseignement supérieur et à ses diplômes constitue une stratégie privilégiée pour atteindre cet objectif, les parents scolarisés sont mieux outillés pour structurer le parcours scolaire de leurs enfants (Hao et Bonstead-Bruns, 1998). En dépit des progrès indéniables reconnus au Québec et, plus largement au Canada, il existe toujours d'importantes disparités de réussite entre les immigrants selon l'origine géographique des parents. Ces disparités peuvent prendre différentes formes : inégalité d'accès, inégalité de persévérance et de diplomation ou plus globalement inégalités de parcours scolaires. Cet article s'intéresse à cette dernière forme de disparités scolaires.

\section{Systèmes éducatifs et variabilité des parcours scolaires}

Tous les systèmes éducatifs sont organisés par cycles d'enseignement, lesquels sont eux-mêmes divisés en filières et en programmes d'études. Cette organisation structure des cheminements officiels qui peuvent être rigides ou au contraire plus ou moins souples. $\mathrm{Si}$, dans plusieurs sociétés développées, le passage du primaire au secondaire est automatique et obligatoire, il n'en est pas ainsi pour le passage entre le secondaire et l'enseignement supérieur. Au contraire, celui-ci est conditionnel, non seulement, à la décision de l'élève, mais également et surtout aux règles institutionnelles régissant la sélection et I'orientation scolaire dans les différentes filières du secondaire. Dans 
plusieurs pays, l'enseignement secondaire est divisé en filières générales et professionnelles. Les premières prescrivent la poursuite des études supérieures et les secondes préparent à la sortie du système scolaire et à l'entrée sur le marché du travail. Comme le notent Picard et al. (2011 : 3), "les systèmes d'éducation prescrivent ainsi un itinéraire type, normalisé et forcément linéaire, qui encadre le parcours scolaire, du primaire à l'enseignement supérieur et jusqu'à l'obtention du diplôme"..

Bien qu'en théorie la sélection et l'orientation scolaire soient fondées sur les compétences et les choix de l'élève, les études menées dans divers contextes sociaux ont démontré que l'affectation des élèves dans ces deux types de filières est davantage le reflet des inégalités sociales par la médiation des performances et des aspirations scolaires (Duru-Bellat et al.2010; Felouzis, 2014). Dès lors que les enfants des familles aisées s'orientent davantage dans les filières générales et ceux des familles modestes dans les filières professionnelles, l'enseignement secondaire devient un espace de segmentation scolaire (Lucas, 2001) et I'enseignement supérieur un instrument de production et de reproduction des inégalités sociales (Alon, 2009). Celles-ci prennent appui sur la mobilisation et l'interaction des différentes formes de capital (culturel, social et économique) qui se jouent à l'avantage des familles de classes moyennes et supérieures (Lynch et O'riordan, 1998). En plus de la valeur qu'elles attribuent aux études supérieures et des ressources financières qu'elles investissent pour que leurs enfants fréquentent les meilleurs établissements et reçoivent la meilleure éducation possible, ces familles possèdent davantage d'information et de savoirs leur permettant d'assurer un encadrement et un suivi structurés qui soutiennent la réussite et la persévérance aux études.

Afin d'atténuer cette forme de ségrégation, qui affecte davantage les élèves de milieux défavorisés et parmi lesquels on compte de nombreuses minorités ethnoculturelles issues de l'immigration, plusieurs pays ont institué des mesures d'assouplissement et de flexibilité dans les conditions d'admission. Par là, on entend la diversification des parcours par différentes formes de bifurcations empruntées par l'étudiant (Picard et al., 2011). Il s'agit, par exemple, d'un changement d'orientation, facilité par les passerelles d'une filière générale à une filière technique ou professionnelle, $d$ 'un retour en arrière vers un cycle moins avancé à des fins de rattrapage ou d'un retour aux études après une période d'interruption (Charbonneau, 2006). Cette flexibilité des structures scolaires permet d'institutionnaliser des parcours traditionnellement considérés comme "atypiques" (Boutinet, 2007) ou "erratiques" (Bloomer et Hodkinson, 2000) par rapport aux parcours prescrits et laisse davantage de place à l'individualisation des cheminements scolaires (Picard et al., 2011).

Une diversification des parcours se fait sentir avec la création de passerelles et d'articulations plus étroites entre l'enseignement secondaire professionnel et l'enseignement supérieur; ce qui conduit à un élargissement des possibilités d'accès au supérieur et ainsi à répondre à une demande sociale accrue de l'enseignement supérieur (Hoelscher et al., 2008; Maclean et Pavlova, 2013). Cette restructuration des systèmes éducatifs a donné lieu à la multiplication des parcours linéaires et non linéaires. Les premiers se distinguent par des cheminements continus ou réguliers prescrits à l'enseignement supérieur et normés à cet effet, alors que les seconds sont discontinus et marqués par des 
bifurcations combinant souvent des programmes de formations professionnelle et générale (Boutinet, 2007). En d'autres termes, les élèves et les étudiants sont autorisés à effectuer des combinaisons de programmes ou de filières différentes au gré de leur volonté. D'une part, cette flexibilité offre aux élèves et étudiants la possibilité de modifier leurs choix et ainsi de réajuster ou réorienter leurs projets académiques et professionnels selon leurs expériences et les temporalités (Bloomer et Hodkinson, 2000). D'autre part, elle offre une chance de rattrapage à ceux que la sélection n'a pas permis de s'inscrire dans un programme ou une filière initialement souhaitée. Dans cette perspective, elle est considérée à juste titre comme un instrument d'amélioration de la démocratisation des études supérieures (Hoelscher et al., 2008; Maclean et Pavlova, 2013).

L'importance des parcours non linéaires et leur effet sur la démocratisation des études supérieures tendent cependant à varier considérablement selon les systèmes éducatifs et les politiques publiques qui les structurent (Kamanzi et al., 2014; Powell et al., 2009). Autrement dit, comme le notent Kamanzi et al. (2017 : 576) : «Rien n'indique qu'a priori cette ouverture à la différenciation est à l'abri de la reproduction sociale, car la diversification des parcours scolaires n'est jamais complètement affranchie de l'emprise de diverses appartenances sociales". Dès lors, cet ancrage "local" des systèmes éducatifs incite à étudier la différenciation des parcours selon l'échelle politique pertinente. Au Canada, cette échelle est essentiellement les provinces qui ont la responsabilité constitutionnelle de l'éducation, mais aussi et surtout les villes où s'installe la grande majorité des immigrants.

\section{Organisation des parcours scolaires au Québec}

Au Québec, la différenciation et la diversification des parcours conduisant aux études supérieures ont été instituées par la réforme Parent (du nom du président de la commission qui en a élaboré le projet, Monseigneur AlphonseMarie Parent) au milieu des années 1960 pour renforcer la démocratisation de l'éducation (Henripin, 1999). Depuis, l'enseignement secondaire a été divisé en deux cycles. Le premier cycle dure deux ans et le deuxième, trois ans. Au cours du deuxième cycle, les élèves peuvent poursuivre le programme général conduisant au diplôme d'études secondaires (DES) ou s'orienter vers la formation professionnelle menant à un diplôme d'études professionnelles (DEP). Toutefois, dans les faits, les élèves ne poursuivent qu'en très petit nombre l'enseignement professionnel, ce que souligne la moyenne d'âge des élèves dans cet enseignement qui est de vingt-six ans. Il existe aussi des programmes pour les élèves en difficultés scolaires qui conduisent à des qualifications. Ces élèves peuvent aussi poursuivre leurs études en formation générale des adultes. Ainsi, il existe une différenciation possible des parcours au sein même du secondaire.

Après les études secondaires, les élèves qui souhaitent poursuivre les études supérieures doivent faire une demande d'admission dans un collège d'enseignement général et professionnel (CEGEP), premier palier de l'enseignement supérieur servant d'intermédiaire entre le secondaire et I'université. Dans le contexte de l'Amérique du Nord, c'est l'équivalent du community college aux États-Unis et dans le reste du Canada. Toutefois, à la 
différence de ce dernier, le CEGEP n'est pas parallèle à l'université, mais un palier d'orientation inférieur et conditionnel à celle-ci. En théorie, le DES et le DEP sont équivalents, mais ils ne le sont pas dans les faits, car les diplômés de la formation professionnelle qui désirent continuer les études au CEGEP sont obligés de suivre et réussir des cours complémentaires du programme de formation générale pour obtenir les prérequis exigés par le CEGEP. II en résulte que deux profils d'étudiants accèdent aujourd'hui au CEGEP : les diplômés du secondaire général dont les parcours sont linéaires et ceux ayant une double formation (professionnelle et générale) dont les parcours sont plutôt non linéaires et sont surtout le fait d'étudiants adultes en retour aux études. Par ailleurs, le CEGEP est lui-même divisé en deux : la voie préuniversitaire et la voie technique. La première dure deux ans et prépare l'entrée aux études universitaires comme son nom l'indique. Quant à la seconde, elle dure trois ans et dispense une formation professionnelle destinée à des futurs cadres moyens et techniciens supérieurs. Les deux formations donnent accès à l'université.

\section{Figure 1 : Organisation du système scolaire du Québec}

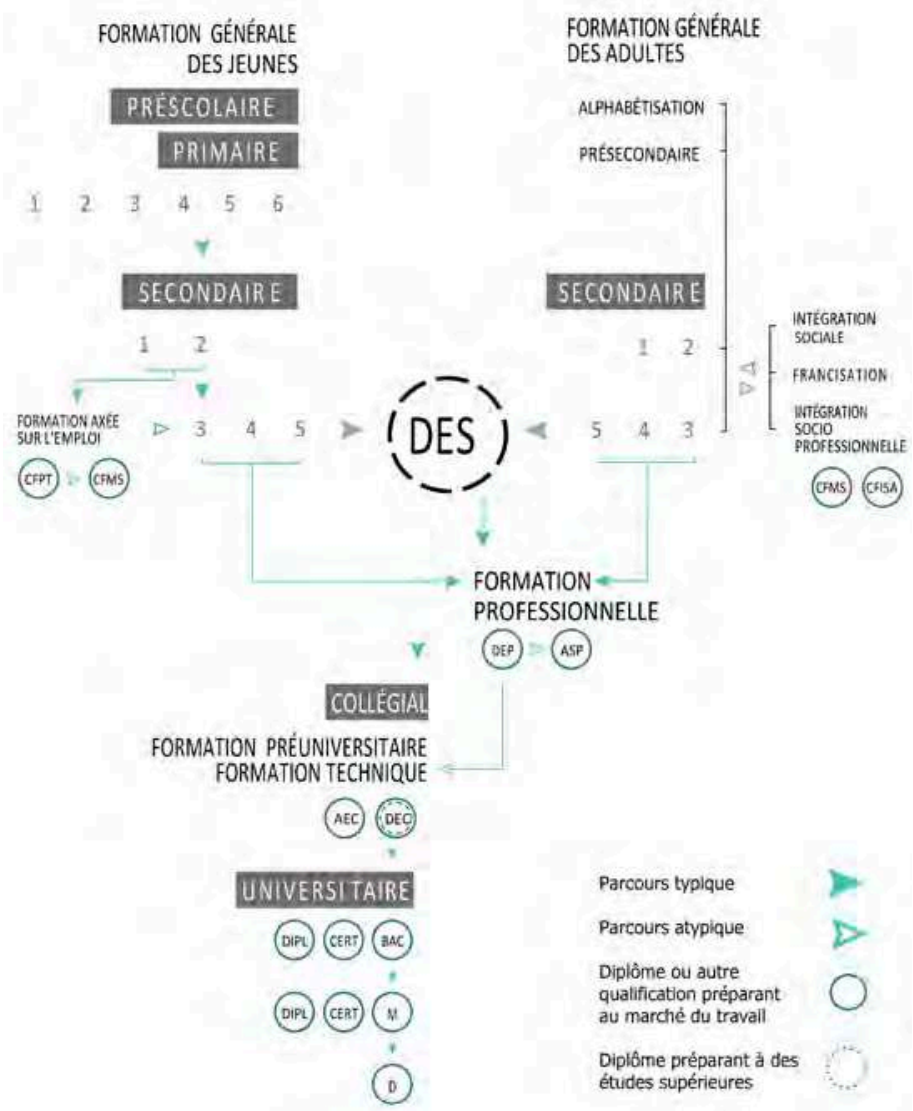

Source : Ministère de l'Éducation, du Loisir et du Sport (http://www.education.gouv.qc.ca/ references/publications/resultats-de-la-recherche/detail/article/etudier-au-quebec/) 


\section{Méthodologie}

\section{Sources des données et échantillon}

La plupart des données utilisées dans cet article ont été fournies par le ministère de l'Éducation du Québec à partir du fichier des données administratives. L'échantillon initial est composé de 47749 sujets faisant partie de deux cohortes d'élèves entrés en première année du secondaire respectivement en 1994-1995 ( $n=23665$ ) et en 2002-2003 ( $n=24084)$ et observées chacune sur une période de dix ans. La première cohorte est entrée au secondaire un peu avant la mise en œuvre de la réforme "Renouveau pédagogique" en 1996-1997, la deuxième la plus importante dans I'histoire du Québec après celle mise en place en 1964, connue sous le nom de Réforme Parent. Quant à la seconde cohorte, elle est la première génération de cette réforme. Le choix de ces deux cohortes vise à tenir compte de l'effet de cette réforme qui visait principalement à accroître la démocratisation de l'éducation par l'amélioration de la réussite à tous les niveaux d'enseignement et l'accessibilité des études supérieures. Parmi ses stratégies, la réforme met l'accent sur l'individualisation et la diversification des parcours, notamment au secondaire, de manière à réduire le taux de décrochage scolaire. Pour chacune des deux cohortes, le sous-échantillon représente $25 \%$ de l'effectif total des élèves.

Selon le lieu de résidence, I'analyse montre qu'environ $95 \%$ des élèves d'origine immigrante faisant partie de cet échantillon habitaient dans la ville de Montréal et ses banlieues. Comme l'étude consiste à comparer les immigrants aux non-immigrants, I'analyse se limitera aux élèves/étudiants dont les parents résidaient dans cette région afin d'éviter le biais du contexte géographique et socioéconomique. Le sous-échantillon retenu est composé de 20387 élèves/ étudiants, dont 5334 (26\%) ont au moins un parent immigrant de première génération, c'est-à-dire né à l'étranger. Le tableau 1 dresse le profil général du sous-échantillon.

Tableau 1 : Répartition des sujets selon l'origine géographique des parents

\begin{tabular}{lcccccc}
\hline & \multicolumn{2}{c}{ Cohorte } & $1994-1995$ & \multicolumn{2}{c}{ Cohorte $2002-2003$} & \multicolumn{2}{c}{ Total } \\
& $\mathrm{N}$ & $\%$ & $\mathrm{~N}$ & $\%$ & $\mathrm{~N}$ & $\%$ \\
\hline Canada & 7188 & 75,18 & 7865 & 72,14 & 15053 & 73,84 \\
Mixte & 679 & 7,16 & 999 & 9,16 & 1678 & 8,23 \\
Asie de I'Est & 94 & 0,99 & 117 & 1,07 & 211 & 1,03 \\
Asie du Sud-Est & 286 & 3,02 & 370 & 3,39 & 656 & 3,22 \\
Afrique du Nord & 312 & 3,29 & 450 & 4,13 & 762 & 3,74 \\
Afrique subsaharienne & 48 & 0,51 & 110 & 1,01 & 158 & 0,77 \\
Europe & 388 & 4,09 & 349 & 3,20 & 737 & 3,62 \\
Amérique latine et Caraïbes & 490 & 5,17 & 642 & 5,89 & 1132 & 5,55 \\
Tous & 9485 & 100,00 & 10902 & 100,00 & 20387 & 100,00 \\
\hline
\end{tabular}

Source : Ministère de l'Éducation du Québec ; calcul des auteurs.

Comme il s'agit des données extraites des dossiers des élèves et étudiants et non d'une enquête auprès de ces derniers ni de leurs parents, elles sont limitées sur le plan méthodologique. Ainsi, la base ne contient pas d'information sur certains facteurs structurant les parcours des élèves et étudiants : par exemple, 
les expériences scolaires telles que perçues ou vécues par les élèves, les rapports entre ces derniers et divers acteurs scolaires (le personnel des établissements scolaires, les parents et autres). En raison de la confidentialité, la base de données ne contient pas non plus de renseignement sur les résultats scolaires de l'élève. Pour combler certaines lacunes, nous avons complété la base de données par certains renseignements disponibles dans les recensements canadiens de 2001 et 2006 permettant de caractériser l'environnement socioéconomique dans lequel évolue l'élève. Dans le cadre de cet article, il s'agit du revenu médian des ménages résidant dans le quartier de résidence, ainsi que le pourcentage des diplômés d'universités.

Pour chaque cohorte, la base de données contient des données longitudinales permettant d'étudier les parcours scolaires des étudiants sur une période de dix ans au moment où la majorité est âgée de vingt-deux ans, c'est-à-dire jusqu'en 2004-2005 pour la première cohorte et en 2012-2013 pour la seconde. Elle contient en outre des renseignements sur certaines caractéristiques sociodémographiques (genre, pays d'origine des parents) et scolaires (type d'établissement fréquenté à chaque palier d'orientation, la présence ou non de retard scolaire) permettant d'analyser ces parcours.

\section{Définition des variables étudiées}

La variable dépendante au cœur de cet article est le parcours scolaire aux études supérieures (CEGEP ou université). Elle a été mesurée par le cheminement aux études pendant et après la scolarité secondaire. La base de données utilisée permet d'identifier les élèves qui ont poursuivi les études au CEGEP et, parmi eux, ceux qui ont, par la suite, fréquenté l'université. En outre, il est possible de distinguer ceux qui ont emprunté des parcours linéaires de ceux qui ont emprunté des parcours non linéaires pour accéder au CEGEP. Les parcours linéaires comprennent les étudiants qui, après l'obtention du diplôme d'études secondaires, ont entrepris des études au CEGEP, qu'ils aient poursuivi ou non à l'université. À l'inverse, les parcours non linéaires comprennent ceux qui, après l'obtention de ce diplôme, se sont inscrits dans un programme parallèle ou équivalent. II s'agit des élèves qui, après l'obtention du diplôme d'études secondaires professionnelles, ont entrepris des études dans un programme général et vice versa. Suivant ces critères, les sujets de notre échantillon sont regroupés en quatre catégories d'élèves/d'étudiants :

- Non-accès aux études supérieures : ceux qui, après les études secondaires, ont mis fin à leur scolarité, du moins pour la période d'observation;

- Parcours linéaire terminal au CEGEP : ceux qui, après I'obtention d'un diplôme d'études secondaires en formation générale, ont continué les études au CEGEP technique ou préuniversitaire, mais n'ont pas poursuivi d'études universitaires; - Parcours linéaire à I'université : ceux qui, après une formation générale au secondaire, sont allés au CEGEP et ont, par la suite, poursuivi leurs études à I'université;

- Parcours non linéaire : ceux qui, avant de s'inscrire dans un CEGEP, ont accompli une double formation au secondaire (générale et professionnelle).

La variable indépendante est l'origine géographique des parents de l'élève ou de l'étudiant. Elle a été mesurée par le pays de naissance des deux parents. Afin d'obtenir des effectifs suffisants pour l'analyse, les pays ont été regroupés 
par région géographique. En nous inspirant des catégorisations utilisées dans quelques études antérieures (Finnie et Mueller, 2008; Kamanzi et al., 2016 ; Thiessen, 2009) et en tenant compte du nombre de sujets par pays et région d'origine des parents, les élèves et étudiants ont été regroupés en huit catégories : (1) Canada ; (2) mixte, I'un des deux parents est immigrant et l'autre canadien de naissance ; (3) Europe et pays anglo-saxons ; (4) Asie orientale ; (5) Asie du Sud-Est et Îles du Pacifique ; (6) Afrique du Nord et Moyen-Orient ; (7) Afrique subsaharienne ; et (8) Amérique latine et Caraïbes. Le tableau 1 décrit la distribution de l'échantillon par rapport à cette variable.

Les variables de contrôle. Trois variables liées à la scolarité secondaire dont les analyses préliminaires ont révélé une influence significative sur les parcours scolaires ont été incluses dans les analyses: le type d'établissement secondaire fréquenté (privé ou public) ; I'âge à l'entrée au secondaire indiquant si l'élève a connu ou non un retard scolaire; et la difficulté d'adaptation scolaire ou d'apprentissage. Le dossier de l'élève contient un code précisant si l'élève a eu des difficultés d'adaptation ou d'apprentissage scolaire, de même que l'âge auquel il est entré en première année au secondaire. En outre, deux variables sociodémographiques seront également prises en compte : le sexe et l'origine socioéconomique de l'élève. Cette dernière est mesurée par deux indicateurs en lien avec les caractéristiques de la localité habitée par les parents de l'élève (unité de recensement) : le pourcentage des diplômés d'université et le revenu médian par ménage. Rappelons que ces deux variables ont été créées à partir des données du recensement canadien de 2001 dans le cas de la cohorte 1994-1995 et du recensement de 2006 dans le cas de la cohorte de 2002-2003.

\section{Modèle d'analyse statistique}

Dans un premier temps, des analyses descriptives ont été effectuées pour dégager le portrait global des parcours scolaires aux études supérieures selon la variable indépendante. Par la suite, des analyses logistiques multinomiales (Allison, 2003) tenant compte d'autres variables associées aux caractéristiques sociodémographiques et scolaires ont été effectuées pour estimer l'influence relative de cette dernière. Le choix de ce modèle est justifié par le fait que la variable dépendante est catégorielle.

Quatre probabilités associées aux catégories de cette variable sont comparées : (1) $p(y=0)$ : non-accès aux études supérieures; $(2) p(y=1)$ : parcours linéaire terminal au CEGEP; (3) p $(y=2)$ : parcours linéaire jusqu'à l'université; et (4) $p(y=3)$ : parcours non linéaire au CEGEP. Sur le plan statistique, le but de l'analyse est d'estimer l'influence de l'origine géographique des parents de l'étudiant sur la probabilité d'emprunter l'un ou l'autre de ces parcours sous l'effet des facteurs $X_{1}, X_{2}, \ldots X_{k}$. La modélisation par la fonction logit permet ainsi de comparer la probabilité associée à chacun de ces parcours à celle de l'inverse, c'est-à-dire, ne pas accéder aux études supérieures ou $\mathrm{p}(\mathrm{y}=0)$. Cette comparaison se résume en trois équations : 


$$
\begin{aligned}
& \operatorname{logit}\left[p\left(y=1 \mid X_{1}, X_{2}, \ldots X_{k}\right)\right]=\ln \frac{p\left(y=1 \mid X_{1}, X_{2} \ldots X_{k}\right)}{p\left(y=0 \mid X_{1}, X_{2} \ldots X_{k}\right)}=\alpha_{1}+\beta_{11} \mathrm{X}_{1}+\beta_{21} \mathrm{X}_{2}+\ldots+\beta_{\mathrm{k} 1} \mathrm{X}_{\mathrm{k}}(1), \\
& \operatorname{logit}\left[p\left(y=2 \mid X_{1}, X_{2}, \ldots X_{k}\right)\right]=\ln \frac{p\left(y=2 \mid X_{2}, X_{2} \ldots X_{k}\right)}{p\left(y=0 \mid X_{2}, X_{2} \ldots X_{k}\right)}=\alpha_{2}+\beta_{12} \mathrm{X}_{1}+\beta_{22} \mathrm{X}_{2}+\ldots+\beta_{\mathrm{k} 2} \mathrm{X}_{\mathrm{k}}(2), \\
& \operatorname{logit}\left[p\left(y=3 \mid X_{1}, X_{2}, \ldots X_{k}\right)\right]=\ln \frac{p\left(y=3 \mid X_{1}, X_{2} \ldots X_{k}\right)}{p\left(y=0 \mid X_{2}, X_{2} \ldots X_{k}\right)}=\alpha_{3}+\beta_{13} \mathrm{X}_{1}+\beta_{23} \mathrm{X}_{2}+\ldots+\beta_{\mathrm{k} 3} \mathrm{X}_{\mathrm{k}}(3),
\end{aligned}
$$

Où $\alpha_{1}, \alpha_{2}$ et $\alpha_{3}$ représentent l'ordonnée à l'origine ou la constante respectivement pour les équations (1), (2) et (3), tandis que $\beta_{1,}, \beta_{2,}, \ldots, \beta_{k}$. représentent les coefficients de régression respectivement associés aux facteurs $X_{1}, X_{2}, \ldots X_{k}$ pour les mêmes équations.

Afin de faciliter l'interprétation, les coefficients de régression ont été transformés en rapports de cotes $(\mathrm{RC})$ ou odds ratios de la manière suivante :

$$
\begin{aligned}
& \mathrm{RC}_{1}=\operatorname{Exp}\left(\alpha_{1}+\beta_{11} \mathrm{X}_{1}+\beta_{21} \mathrm{X}_{2}+\ldots+\beta_{\mathrm{k} 1} \mathrm{X}_{\mathrm{k}}\right)=e^{\alpha_{2}+\beta_{12} X_{1}+\beta_{22} X_{2}+\ldots+\beta_{k 1} X_{\mathrm{k}}} \\
& \mathrm{RC}_{2}=\operatorname{Exp}\left(\alpha_{2}+\beta_{12} \mathrm{X}_{1}+\beta_{22} \mathrm{X}_{2}+\ldots+\beta_{\mathrm{k} 2} \mathrm{X}_{\mathrm{k}}\right)=e^{\alpha_{2}+\beta_{22} X_{2}+\beta_{22} X_{2}+\ldots+\beta_{\mathrm{k} 2} X_{\mathrm{k}}} \\
& \mathrm{RC}_{3}=\operatorname{Exp}\left(\alpha_{3}+\beta_{13} \mathrm{X}_{1}+\beta_{23} \mathrm{X}_{2}+\ldots+\beta_{\mathrm{k} 3} \mathrm{X}_{\mathrm{k}}\right)=e^{\alpha_{3}+\beta_{13} X_{1}+\beta_{23} X_{2}+\cdots+\beta_{\mathrm{kg}} X_{\mathrm{k}}}
\end{aligned}
$$

\section{Des parcours scolaires variés}

Après les études secondaires, la majorité des jeunes Québécois poursuivent des études supérieures. Selon l'échantillon utilisé (Tableau 2), dix ans après I'entrée en première année secondaire, près des deux tiers $(64,92 \%)$ des élèves avaient fréquenté, à un moment ou un autre, un établissement d'études supérieures. Parmi eux, 34,13\% avaient fréquenté un CEGEP et 30,80 \% avaient poursuivi les études jusqu'à l'université. Le taux de fréquentation varie toutefois selon I'origine géographique des parents. II est nettement plus élevé chez les jeunes issus des familles originaires d'Asie orientale $(80,20 \%)$, d'Afrique du Nord et Moyen-Orient (74,03\%), mais relativement moins élevé chez ceux d'Amérique latine et des Caraïbes $(57,77 \%)$.

Outre le plus haut niveau d'études de l'établissement fréquenté (CEGEP ou université), les étudiants se distinguent entre eux par le type de parcours emprunté. Selon l'analyse, 55,92 \% des étudiants empruntent un parcours linéaire $(30,80 \%$ jusqu'à l'université et $25,12 \%$ au CEGEP). En revanche, presque un étudiant sur dix $(9,01 \%)$ accède au CEGEP par la voie d'un parcours non linéaire. Rappelons que cette catégorie d'étudiants est composée d'étudiants qui, après l'obtention d'un diplôme d'études secondaires dans un programme de formation professionnelle, ont suivi des cours dans un programme de formation générale et vice versa. Toutefois, l'analyse supplémentaire révèle que 
la majorité (68 \%) est composée de diplômés de formation professionnelle ayant suivi des cours dans un programme général pour être admissibles au CEGEP.

Tableau 2 : Répartition des sujets selon l'origine géographique des parents et le type de parcours scolaire aux études supérieures (ÉS)

\begin{tabular}{lcccccc}
\hline & $\begin{array}{c}\text { Non-accès } \\
\text { aux ÉS } \\
\text { (parcours 0) }\end{array}$ & $\begin{array}{c}\text { Accès } \\
\text { aux ÉS }\end{array}$ & $\begin{array}{c}\text { Parcours } \\
(1)\end{array}$ & $\begin{array}{c}\text { Parcours } \\
(2)\end{array}$ & $\begin{array}{c}\text { Parcours } \\
(3)\end{array}$ \\
& $\%$ & $\%$ & $\%$ & $\%$ & $\%$ & N \\
\hline Canada & 35,35 & 64,65 & 25,31 & 29,59 & 9,75 & 14594 \\
Un seul parent né au Canada & 35,81 & 64,19 & 24,04 & 33,64 & 6,51 & 1614 \\
Asie orientale & 19,80 & 80,20 & 20,30 & 57,92 & 1,98 & 202 \\
Asie du Sud-Est et Îles du Pacifique & 32,18 & 67,82 & 25,55 & 38,17 & 4,10 & 634 \\
Afrique du Nord et Moyen-Orient & 25,93 & 74,03 & 21,93 & 44,83 & 7,31 & 725 \\
Afrique subsaharienne & 36,30 & 63,70 & 23,97 & 33,56 & 6,16 & 146 \\
Europe/pays anglo-saxons & 33,19 & 66,81 & 23,57 & 36,40 & 6,83 & 717 \\
Amérique latine et Caraïbes & 42,23 & 57,77 & 19,70 & 28,22 & 9,85 & 1056 \\
Tous & 35,07 & 64,92 & 25,12 & 30,80 & 9,01 & 19688 \\
\hline
\end{tabular}

\author{
Parcours (0) : parcours qui se terminent au secondaire \\ Parcours (1) : linéaire secondaire $\Rightarrow$ CEGEP \\ Parcours (2) : linéaire secondaire $\Rightarrow$ CEGEP $\Rightarrow$ université \\ Parcours $(3)$ : non linéaire secondaire $\Rightarrow$ CEGEP \\ Source : Ministère de l'Éducation du Québec ; calcul des auteurs.
}

Alors que l'engagement dans un parcours linéaire ou non linéaire relève théoriquement des choix de l'étudiant, les résultats révèlent d'importantes différences selon I'origine géographique des parents. Dans l'ensemble, à I'exception de ceux qui viennent d'Amérique latine et des Caraïbes (9,85\%), les étudiants issus des parents immigrants sont moins enclins à emprunter les parcours non linéaires, comparativement à leurs pairs dont les deux parents sont natifs du Canada (9,75\%). Ainsi, leur proportion est très faible chez ceux dont les parents viennent des pays de I'Asie orientale (1,98\%), de même que chez ceux de I'Asie du Sud-Est et des Îles-du-Pacifique (4,10\%). À I'inverse, les élèves des familles immigrantes originaires de ces régions s'inscrivent dans les parcours linéaires jusqu'à l'université dans une proportion relativement plus élevée que leurs pairs issus des parents nés au Canada (29,59\%), soit 57,92\% chez ceux originaires d'Asie orientale, 44,83\% chez ceux de l'Afrique du Nord et du Moyen-Orient, 38,27 \% chez ceux de I'Asie du Sud-Est, 36,40 \% chez ceux de I'Europe et des pays anglo-saxons et $34 \%$ pour l'Afrique subsaharienne. La situation semble toutefois globalement comparable pour le parcours linéaire aux études collégiales sans poursuite à l'université, du moins pour la période d'observation. L'analyse montre qu'il y a relativement peu de différences à ce niveau. 


\section{Des choix de parcours fortement modulés par les facteurs scolaires et extrascolaires}

Les analyses multinomiales (Tableau 3) permettent d'examiner si les différences de parcours observées selon l'origine géographique des parents sont statistiquement significatives et dans quelle mesure elles sont attribuables à cette variable ou à l'effet des antécédents scolaires et des caractéristiques sociodémographiques des élèves. Le modèle 1 inclut seulement l'origine géographique des parents de l'élève, en distinguant les immigrants de première génération de ceux de deuxième génération. Les résultats précisent ceux de l'analyse descriptive précédente (Tableau 2), indiquant la présence de différences significatives en ce qui a trait aux parcours linéaires et, dans une moindre mesure, aux parcours non linéaires. De façon globale, les enfants d'immigrants sont davantage enclins à emprunter les parcours linéaires; à l'inverse, ils sont moins susceptibles de s'engager dans des parcours non linéaires.

En ce qui concerne les parcours linéaires, les résultats permettent de dégager trois principaux constats. Premièrement, les différences entre les étudiants issus de l'immigration et les autres sont nettement plus élevées à I'université qu'au CEGEP. Deuxièmement, la situation est fortement variable au sein des immigrants eux-mêmes. Troisièmement, des différences significatives s'observent principalement auprès des immigrants de deuxième génération, alors qu'elles sont relativement faibles pour ceux de la première génération surtout au CEGEP. Ainsi, par rapport aux non-immigrants, la probabilité de suivre ce parcours est de neuf fois $(8,96)$ plus élevée chez les immigrants de deuxième génération originaire d'Asie orientale et presque quatre fois chez ceux originaires d'Asie du Sud-Est et des Îles du Pacifique $(3,56)$, d'Afrique du Nord et Moyen-Orient $(3,79)$. À noter cependant que les enfants d'immigrants originaires d'Amérique latine et des Caraïbes font l'exception : ils sont moins susceptibles de suivre les parcours linéaires jusqu'à l'université, comparativement aux non-immigrants $(0,89)$.

En ce qui a trait aux parcours non linéaires, la situation est quelque peu inversée, comme souligné précédemment : à l'exception de ceux qui sont originaires de l'Afrique subsaharienne, les immigrants sont moins susceptibles d'emprunter ces parcours. À cet égard, l'analyse permet de dégager deux observations. Les différences significatives observées sont encore une fois très variables selon l'origine géographique des parents de l'élève, mais se rapportent cette fois-ci aux immigrants de première génération, alors que la situation est plutôt comparable entre ceux de deuxième génération et les non-immigrants. 
Tableau 3 : Coefficients de régression multinomiale

\begin{tabular}{|c|c|c|c|c|c|c|}
\hline & \multicolumn{3}{|c|}{ Modèle 1} & \multicolumn{3}{|c|}{ Modèle 2} \\
\hline & $\begin{array}{l}\text { Parcours } \\
\text { (1) }\end{array}$ & $\begin{array}{l}\text { Parcours } \\
\text { (2) }\end{array}$ & $\begin{array}{l}\text { Parcours } \\
\text { (3) }\end{array}$ & $\begin{array}{l}\text { Parcours } \\
\quad(1)\end{array}$ & $\begin{array}{l}\text { Parcours } \\
\quad(2)\end{array}$ & $\begin{array}{l}\text { Parcours } \\
\text { (3) }\end{array}$ \\
\hline & $\operatorname{Exp}(b)$ & $\operatorname{Exp}(b)$ & $\operatorname{Exp}(b)$ & $\operatorname{Exp}(b)$ & $\operatorname{Exp}(b)$ & $\operatorname{Exp}(b)$ \\
\hline \multicolumn{7}{|c|}{ Variable indépendante : origine des parents } \\
\hline Canada (référence) & 1,00 & 1,00 & 1,00 & 1,00 & 1,00 & 1,00 \\
\hline Un seul parent né au Canada & $0,94(\mathrm{~ns})$ & 1,12 (ns) & $0,66 * * *$ & $1,01(\mathrm{~ns})$ & 1,10 (ns) & $0,69 * *$ \\
\hline \multicolumn{7}{|l|}{ Asie de l'Est } \\
\hline 2e génération & $3,84^{*}$ & $8,96 * * *$ & $2,71(\mathrm{~ns})$ & $5,27 * *$ & $12,06 * * *$ & 3,41 (ns) \\
\hline 1e génération & $1,16(\mathrm{~ns})$ & $2,89 * * *$ & $0,10^{*}$ & $2,05^{*}$ & $5,09 * * *$ & $0,16(\mathrm{~ns})$ \\
\hline \multicolumn{7}{|l|}{ Asie du Sud-Est et Îles du Pacifique } \\
\hline 2e génération & $2,25 * * *$ & $3,56 * * *$ & 1,07 & $2,66 * * *$ & $4,00 * * *$ & 1,15 (ns) \\
\hline 1e génération & $0,70^{*}$ & $0,64 * *$ & $0,24 * * *$ & $2,13^{* * *}$ & $2,70 * * *$ & 0,55 (ns) \\
\hline \multicolumn{7}{|l|}{ Afrique du Nord et Moyen-Orient } \\
\hline 2e génération & $1,64^{*}$ & $3,79 * * *$ & $1,63^{*}$ & $1,63^{*}$ & $2,79 * * *$ & $1,57 \mathrm{~ns})$ \\
\hline 1e génération & $1,01(\mathrm{~ns})$ & $1,42 * *$ & 0,79 (ns) & $1,71 * * *$ & $2,48 * * *$ & 1,20 (ns) \\
\hline \multicolumn{7}{|l|}{ Afrique subsaharienne } \\
\hline 2e génération & 1,40 (ns) & $3,98 * * *$ & $4,22^{*}$ & 2,00 (ns) & $5,21 * *$ & $5,16^{* *}$ \\
\hline 1e génération & $0,86(\mathrm{~ns})$ & $0,74(\mathrm{~ns})$ & $0,15^{*}$ & $1,92 *$ & $1,87^{*}$ & 0,26 (ns) \\
\hline \multicolumn{7}{|l|}{ Europe/pays anglo-saxons } \\
\hline 2e génération & $1,29(\mathrm{~ns})$ & $1,43^{* *}$ & $0,82(\mathrm{~ns})$ & $1,55^{* *}$ & $1,74 * * *$ & 1,06 (ns) \\
\hline 1e génération & $0,72 *$ & $1,19(\mathrm{~ns})$ & $0,68(\mathrm{~ns})$ & $1,09(\mathrm{~ns})$ & $1,58 * *$ & 0,95 (ns) \\
\hline \multicolumn{7}{|l|}{ Amérique latine et Caraïbes } \\
\hline 2e génération & $1,41 * *$ & $0,89 * * *$ & $1,16(n s)$ & $2,06 * * *$ & $1,25(\mathrm{~ns})$ & $1,45^{*}$ \\
\hline 1e génération & $0,55 * * *$ & $0,29 * * *$ & $0,60 * *$ & $1,31 *$ & $0,85(\mathrm{~ns})$ & 1,15 (ns) \\
\hline \multicolumn{7}{|l|}{ Variables de contrôle } \\
\hline \multicolumn{7}{|l|}{ Antécédents scolaires } \\
\hline Difficulté scolaire (oui) & - & - & - & $0,25 * * *$ & $0,10 * * *$ & $0,38 * * *$ \\
\hline $\begin{array}{l}\text { Âge d'entrée en } 1 \mathrm{e} \\
\text { secondaire }\end{array}$ & - & - & - & $0,43 * * *$ & $0,26 * * *$ & $0,62^{* * *}$ \\
\hline $\begin{array}{l}\text { Type d'établissement } \\
\text { secondaire (privé) }\end{array}$ & - & - & - & $2,47 * * *$ & $4,56 * * *$ & $1,86^{* * *}$ \\
\hline \multicolumn{7}{|c|}{ Caractéristiques sociodémographiques } \\
\hline Revenu annuel médian & - & - & - & $1,15^{* * *}$ & $1,16 * * *$ & $1,07^{*}$ \\
\hline \% diplômés universitaires & - & - & - & $1,09 * * *$ & $1,34 * * *$ & $0,92 *$ \\
\hline Sexe (homme) & - & - & - & $0,54 * * *$ & $0,44 * * *$ & $0,80 * * *$ \\
\hline \multicolumn{7}{|l|}{ Cohorte } \\
\hline 1994-1995 (référence) & - & - & - & 1,00 & 1,00 & 1,00 \\
\hline $2002-2003$ & - & - & - & $0,87^{* * *}$ & $1,12 * *$ & $1,54 * * *$ \\
\hline Pseudo- $R^{2}$ & & 0,010 & & & 0,123 & \\
\hline$\chi_{\mathrm{RV}}^{2}$ & & $491,19 * * *$ & & & $6073,16^{* * *}$ & \\
\hline D.L. & & 39 & & & 60 & \\
\hline $\mathrm{N}$ & & 19688 & & & 19097 & \\
\hline
\end{tabular}

Parcours (1) : linéaire secondaire $\Rightarrow$ CEGEP

Parcours (2) : linéaire secondaire $\Rightarrow$ CEGEP $\Rightarrow$ université

Parcours (3) : non linéaire secondaire $\Rightarrow$ CEGEP

${ }^{*} \mathrm{p}<0,05 ;{ }^{* *} \mathrm{p}<0,01 ;{ }^{* * *} \mathrm{p}<0,001 ; \mathrm{RV}$ : rapport de vraisemblance;

D.L. : Nombre de degrés de liberté.

Source : Ministère de l'Éducation du Québec ; calcul des auteurs. 
Le modèle 2 tient compte des variables en lien avec les caractéristiques scolaires et sociodémographiques de l'élève. Les résultats révèlent que ces variables exercent une influence significative sur l'accès aux études supérieures et le choix du parcours. Les élèves ayant connu un retard avant d'entrer au secondaire ou des difficultés scolaires au secondaire, de même que ceux qui ont exclusivement fréquenté un établissement public, ont nettement moins de chances d'accéder aux études supérieures, que ce soit par la voie d'un parcours linéaire ou non linéaire. II en est de même pour les garçons : ils sont moins susceptibles que les filles de poursuivre des études supérieures, que ce soit par la voie des parcours linéaires ou non linéaires. Enfin, plus le revenu annuel médian des ménages et le pourcentage des diplômés résidant dans la zone habitée par les parents de l'élève augmentent, plus la probabilité de suivre un parcours linéaire augmente, surtout à l'université.

L'introduction de ces variables de contrôle modifie sensiblement l'influence de l'origine géographique des parents de l'élève. À caractéristiques scolaires et sociodémographiques comparables, les jeunes issus de l'immigration sont nettement plus susceptibles de poursuivre des études supérieures à l'université comme au CEGEP par la voie des parcours linéaires, qu'ils soient de première ou de deuxième génération. Une analyse supplémentaire révèle toutefois que cette modification de résultats est davantage attribuable aux antécédents scolaires qu'aux caractéristiques sociodémographiques de l'élève. En revanche, les différences tendent à disparaitre en ce qui a trait aux parcours non linéaires Iorsqu'on tient compte de ces variables. Ces résultats appuient ainsi l'hypothèse selon laquelle la poursuite des études supérieures et les parcours empruntés par les jeunes Québécois issus de l'immigration sont fortement influencés par la qualité de leur scolarité au primaire et au secondaire.

\section{Discussion}

Cette étude montre qu'au Québec, les élèves issus de l'immigration poursuivent les études supérieures dans une proportion égale, voire plus élevée que ceux dont les parents sont nés au Canada. Elle confirme ainsi les conclusions des études antérieures qui soulignent la situation exceptionnelle du Canada et du Québec en particulier. Comme mentionné dans la première section, cet accès accru des immigrants à l'enseignement supérieur a été attribué aux politiques d'immigration canadiennes. D'une part, en fondant la sélection des immigrants sur les critères de niveau de scolarisation et de qualification professionnelle, ces politiques favorisent le choix des immigrants ayant les habiletés d'intégration socioéconomique. L'effet significatif positif de la variable visant à tenir compte du capital culturel (pourcentage des diplômés d'universités) dans le quartier de résidence des parents souligne bien le rôle indirect des politiques de sélection des immigrants. Ainsi, on peut aussi penser que les immigrants plus scolarisés assurent un meilleur encadrement des études à leurs enfants (Abada et Tenkorang, 2009), leur transmettent des dispositions stratégiques qui favorisent la réussite et la persévérance scolaire et modulent vers le haut les aspirations scolaires et professionnelles (Ichou, 2014; Thiessen, 2009). La littérature internationale montre que ces capitaux favorisent le choix scolaire et la persévérance jusqu'au supérieur (Douglass et Thomson, 2010). D'autre part, les études reconnaissent aussi qu'au plan national et local, 
cette sélection est arrimée à des mesures visant à favoriser l'intégration des immigrants et à éliminer la ségrégation ou, à tout le moins, à la réduire (Berry, 2013). II nous a été cependant impossible de cerner l'effet de ces mesures sur l'intégration des parents. Les études mentionnent les services et ressources (par ex., le soutien dans l'apprentissage de la langue d'enseignement - français ou anglais) accordés aux enfants nouvellement arrivés au Canada (Shakya et al., 2010), ainsi que les activités visant à promouvoir la collaboration entre les établissements scolaires, les parents et communautés immigrantes (Farmer et Labrie, 2008; Potvin et al., 2016).

Toutefois, à l'instar d'autres recherches antérieures, cette étude reconnaît les limites des politiques d'immigration et l'existence probable de ségrégations à l'endroit de certaines communautés immigrantes qui se manifestent par des parcours scolaires plutôt difficiles et fragiles. Ainsi, comme nous l'avons montré, le fait que les élèves issus des familles immigrées des Caraïbes et de l'Amérique latine accèdent moins aux études supérieures est dû en partie aux antécédents scolaires. Des analyses supplémentaires (Tableau 4) montrent que ces élèves sont moins susceptibles de fréquenter l'école privée. À l'inverse, ils sont proportionnellement plus nombreux à avoir connu des épisodes de difficulté d'apprentissage ou d'adaptation scolaire. Alors que leur taux de décrochage est plus élevé par rapport aux autres (Mc Andrew et al., 2015; Mc Andrew et al., 2013), la base de données qui nous a été fournie contient uniquement les élèves sortis avec un diplôme ou une qualification. La présence des décrocheurs dans l'analyse aurait probablement mis en évidence des différences plus élevées entre ces élèves et les autres quant aux parcours linéaires aux études supérieures. Autrement dit, la non-prise en compte de cette catégorie de variables porte le risque de surestimer leurs parcours aux études supérieures. Dans la même veine, la base de données utilisée ne contient pas d'information sur les résultats scolaires qui conditionnent également les parcours et l'accès aux études supérieures.

Tableau 4 : Caractéristiques de la scolarité au secondaire selon l'origine géographique des parents (\%)

\begin{tabular}{lcccc}
\hline & $\begin{array}{c}\text { A fréquenté un } \\
\text { établissement privé }\end{array}$ & $\begin{array}{c}\text { Retard } \\
\text { scolaire }\end{array}$ & $\begin{array}{c}\text { Difficultés d'adaptation } \\
\text { scolaire et d'apprentissage }\end{array}$ & N \\
\hline Canada & 26,57 & 16,59 & 10,99 & 14594 \\
Mixte & 25,27 & 16,51 & 12,51 & 1614 \\
Asie de l'Est & 38,86 & 36,97 & 5,69 & 202 \\
Asie du Sud-Est & 15,55 & 37,80 & 8,84 & 634 \\
Afrique du Nord & 35,56 & 30,31 & 9,06 & 725 \\
Afrique subsaharienne & 13,92 & 41,77 & 17,72 & 146 \\
Europe & 26,59 & 28,49 & 10,31 & 717 \\
Amérique latine et Caraïbes & 19,43 & 44,17 & 17,76 & 1056 \\
Tous & 26,08 & 20,15 & 11,32 & 19688 \\
\hline
\end{tabular}

Source : Ministère de l'Éducation du Québec ; calcul des auteurs.

Au final, l'étude permet de tirer deux conclusions principales. La première est que la différenciation des parcours contribue à réduire les inégalités devant l'enseignement supérieur. Les résultats montrent que $9,01 \%$ des jeunes 
Québécois empruntent les parcours non linéaires, ce qui représente environ $14 \%$ de ceux qui accèdent au CEGEP. Comme souligné précédemment, ces parcours contribuent notamment à rehausser l'accès aux études supérieures pour les jeunes issus des familles immigrées d'Amérique latine et des Caraïbes, le groupe le moins favorisé à ce sujet : 9,85\% empruntent ces parcours, soit $17 \%$ de ceux qui accèdent au CEGEP. Bien qu'elle demeure modeste, la contribution des parcours non linéaires à l'accès aux études supérieures au Québec est indéniable, comme en témoignent les résultats. Toutefois, l'effet de cette différenciation s'avère limité, car les inégalités se reconstituent et tendent même à se renforcer à l'intérieur de l'enseignement supérieur lui-même à travers les choix de parcours (Duru-Bellat et al., 2010). En d'autres mots, les inégalités quantitatives peuvent se transformer en inégalités qualitatives (Prost, 1992), car "plus d'études ne signifie pas les mêmes études pour tous" (Erlich et Verly, 2010 : 85). Les parcours aux études supérieures auxquels donne lieu cette différenciation sont souvent marqués par une certaine hiérarchie et conduisent à des diplômes de valeur sociale inégale (Erlich et Verly, 2010; Hoelscher et al., 2008). Dès lors, non seulement les politiques de différenciation des parcours doivent être poursuivies pour démocratiser davantage l'accès aux études, mais aussi des mesures d'harmonisation entre ces parcours doivent être pensées pour contrer le risque de la hiérarchie et de la segmentation socioscolaire de l'enseignement supérieur. Les recherches ultérieures devraient se poursuivre dans ce sens pour éclairer les politiques sur les perspectives d'arrimer l'égalité d'accès aux études à l'égalité des résultats. Afin de tenir compte de l'évolution récente des politiques d'immigration aux niveaux provincial et fédéral, elles pourraient également faire des analyses à partir des données plus récentes auxquelles nous n'avons pas eu accès.

\section{Conclusion}

L'objectif de cette étude était de décrire et analyser la morphologie des parcours des jeunes Québécois issus des familles immigrantes dans l'enseignement supérieur, ainsi que les facteurs qui contribuent à leur structuration. Son hypothèse de départ est que ces parcours varient en fonction de l'origine géographique des parents. Pour ce faire, les parcours (linéaires et non linéaires) des différents élèves/étudiants d'origine immigrante ont été comparés à ceux de leurs pairs dont les parents ne sont pas immigrants. Les résultats obtenus à partir des données utilisées permettent de dégager les constats généraux suivants. Premièrement, l'emprunt des parcours linéaires et non linéaires varie de façon statistiquement significative entre les deux catégories d'étudiants. Toutefois, les différences s'avèrent plus élevées dans les parcours linéaires que dans les parcours non linéaires. De plus, en ce qui a trait aux parcours linéaires, I'analyse a mis en évidence que cette différence est nettement plus élevée à l'université qu'au CEGEP. Autrement dit, après le secondaire, les étudiants d'origine immigrante sont nettement plus susceptibles d'enchaîner avec le CEGEP et l'université. En revanche, la différence observée dans les parcours non linéaires est relativement faible et tend même à disparaitre lorsqu'on tient compte des caractéristiques sociodémographiques et scolaires de l'élève. Cela appuie l'hypothèse selon laquelle ces parcours contribuent à la réduction des inégalités et à la démocratisation de l'enseignement supérieur, comme nous l'avons mentionné dans la section dédiée au cadre d'analyse. 
Deuxièmement, il existe des disparités importantes parmi les étudiants issus de l'immigration eux-mêmes selon l'origine géographique de leurs parents et trois groupes d'étudiants se démarquent des autres quant au choix des parcours linéaires jusqu'à l'université : ceux dont les parents viennent de l'Asie orientale; de I'Asie du Sud-Est et des îles du Pacifique ; ainsi que des pays du Maghreb et du Moyen-Orient. Par contre, la situation est inversée pour ceux d'origine caribéenne et latino-américaine. Ces derniers s'inscrivent moins dans ces parcours, comparativement aux autres. Troisièmement, des différences similaires s'observent entre les immigrants de première et deuxième génération au sein des étudiants dont les parents immigrés sont d'une même région géographique. Enfin, quatrièmement, les disparités observées sont en partie attribuables aux antécédents scolaires, lesquels sont eux associés à l'origine des parents et agissent en interaction avec celle-ci dans le choix du parcours.

En somme, les résultats obtenus corroborent ceux des études antérieures, tout en les précisant. Comme nous l'avons mentionné dans la première section, plusieurs études ont révélé que, contrairement à d'autres pays développés, les jeunes canadiens d'origine immigrante accèdent aux études supérieures dans une proportion supérieure à celle de leurs pairs non immigrants (Finnie et Mueller, 2010). Le présent article va cependant plus loin et sa contribution particulière est d'avoir, à partir du concept de parcours, mis en exergue les aspects qualitatifs de ces différences.

\section{Références bibliographiques}

Abada Eric and Tenkorang Eric (2009) Pursuit of university education among the children of immigrants in Canada: The roles of parental human capital and social capital, Journal of Youth Studies, 12 (2), pp. 185-207.

Allison Paul Davis (2003) Logistic Regression. Using the SAS System. Theory and Application, Cary, NC, SAS Institute Inc., 308 p.

Alon Sigal (2009) The evolution of class inequality in higher education: competition, exclusion, and adaptation, American Sociological Review, 74, pp. 731-755.

Auernheimer Georg (2006) The German education system: Dysfunctional for an immigration society, European Education, 37 (4), pp. 75-89.

Baum Sandy and Flores Stella M. (2011) Higher education and children in immigrant families, The Future of Children, 21 (1), pp. 171-193.

Berry John W. (2013) Intercultural relations in plural societies: Research derived from Canadian multiculturalism policy, Canadian Ethnic Studies, 3 (2), pp. 1122-1135.

Bloemraad Irene (2012) Understanding "Canadian Exceptionalism" in Immigration and Pluralism Policy, Washington, DC, Migration Policy Institute, [online] last checked on 31/08/2017. URL: http://www.migrationpolicy.org/ research/TCM-canadian-exceptionalism

Bloomer Martin and Hodkinson Phil (2000) The complexity and unpredictability of young people's learning careers, Education \& Training, 42 (2), pp. 68-74.

Boutinet Jean-Pierre (2007) L'espace contradictoire des conduites à projet : entre le projet d'orientation du jeune et le parcours atypique de l'adulte, L'Orientation scolaire et professionnelle, 36 (1), pp. 19-32. 
Boyd Monica (2002) Educational offspring: success or segmented assimilation?, International Immigration Review, 36 (4), pp. 1036-1060.

Buchmann Claudia and Parrado Emilio A. (2006) Educational achievement of immigrant-origin and native students: A comparative analysis informed by institutional theory, International Perspectives on Education and Society, 7, pp. 335-366.

Charbonneau Johanne (2006) Réversibilité des parcours scolaires au Québec, Cahiers internationaux de sociologie, 20 (1), pp. 111-131.

Crul Maurice, Schnell Phillipp, Herzog-Punzenberger Barbara, Wilmes Maren, Slootman Marieke and Gómez Rosa Aparicio (2012) School careers of second-generation youth in Europe: Which education systems provide the best chances for success?, in Maurice Crul, Jens Schneider et Frans Lelie Eds., The European Second Generation Compared. Does the Integration Context Matter? Amsterdam, Amsterdam University Press, pp. 110-165.

Cutler David Matthew, Glaeser Edward Ludwig and Vigdor Jacob L. (2007) When Are Ghettos Bad? Lessons from Immigrant Segregation in The United States, Working Paper 13082, Cambridge, National Bureau of Economic Research, [online] last checked on 31/08/2017. URL: http://www.nber.org/papers/w13082

Djajic Slobodan (2003) Assimilation of Immigrants: Implications for Human Capital Accumulation of the Second Generation, Journal of Population Economics, 6, pp. 831-845.

Dronkers Jaap and van der Velden Rolf (2013) Positive but also Negative Effects of Ethnic Diversity in Schools on Educational Performance? An EmpiricalTest Using PISA Data, in Michael Windzio Ed., Integration and Inequality in Educational Institutions, New York/London/Dordrecht, Springer Science \& Business Media, pp. 71-98.

Douglass John Aubrey and Thomson Gregg (2010) The immigrant's university: A study of academic performance and the experiences of recent immigrant groups at the University of California, Higher Education Policy, 23 (4), pp. 451-474.

Duru-Bellat Marie, Kieffer Annick et Reimer David (2010) Les inégalités d'accès à l'enseignement supérieur : le rôle des filières êt des spécialités. Une comparaison entre l'Allemagne de l'Ouest et la France, Économie et statistique, 433 (1), pp. 3-22.

Dustmann Christian, FrattiniTommaso and Lanzara Gianandrea (2011) Educational achievement of second generation of immigrant: An international comparison, Discussion paper, London, Centre for Research and Analysis of Migration, Department of Economics, [online] last checked on 31/08/2017. URL: http://www. webmail.norface-migration.org/publ_uploads/NDP_25_11.pdf

Erlich Valérie et Verley Élise (2010) Une relecture sociologique des parcours des étudiants français : entre segmentation et professionnalisation, Éducation et Sociétés : Revue internationale de sociologie de l'éducation, 26 (2), pp. 71-88.

Farmer Diane et Labrie Normand (2008) Immigration et francophonie dans les écoles ontariennes : comment se structurent les rapports entre les institutions, les parents et le monde communautaires?, Revue des sciences de l'éducation, 34 (2), 377-398.

Felouzis Georges (2014) Les inégalités scolaires, Paris, Presses universitaires de France, $128 \mathrm{p}$. 
Felouzis Georges, Liot Françoise et Perroton Joëlle (2005) L'apartheid scolaire : enquête sur la ségrégation ethnique dans les collèges, Paris, Éditions du Seuil, $235 \mathrm{p}$.

Ferrer Ana, Picot Garnett and Riddell W. Craig (2014) New Directions in Immigration Policy: Canada's Evolving Approach to the Selection of Economic Immigrants, International Migration Review, 48 (3), 846-867.

Finnie Ross and Mueller Richard E. (2010) They came, they saw, they enrolled: Access to post-secondary education by the children of Canadian immigrants, in Ross Finnie, Marc Frenette, Richard E. Mueller and Arthur Sweetman Eds., Pursuing higher education in Canada. Economic, social, and policy dimensions, Kingston, Queen's University Press, pp. 192-216.

Finnie Ross et Mueller Richard E. (2008) The effects of family income, parental education and other background factors to postsecondary education in Canada. Measuring the effectiveness of student aid, MESA project paper, Toronto, Educational Policy Institute, 52 p.

Ghosh Ratns (2004) Public Education and multicultural policy in Canada: The special case of Quebec, International Review of Education, 50 (5), pp. 543-566.

Griga Dorit and Hadjar Andreas (2013) Migrant Background and Higher Education Participation in Europe: The Effect of the Educational Systems, European Sociological Review, 30 (3), pp. 275-286.

Hao Linxin and Melissa Bonstead-Bruns (1998) Parent-child differences in Educational expectations and the academic achievement of immigrant and native students, Sociology of Education, 71 (3), pp. 175-198.

Harles John C. (2004) Immigrants integration in Canada and United States, American Review of Canadian Studies, 34 (2), pp. 223-258.

Harles John C. (1997). Integration before assimilation: immigration, multiculturalism and the Canadian polity, Canadian Journal of Political Science/Revue canadienne de science politique, 30, pp. 711-736.

Henripin Marthe (1999) Différencier le curriculum au secondaire : vers des parcours scolaires stimulants pour tous les jeunes : problématique, situation au Québec, défis pour notre système scolaire, Québec, Conseil supérieur de l'éducation, $193 \mathrm{p}$.

Henry Frances and Tator Carol (2010) The colour of democracy: racism in Canadian society, Toronto, Nelson Education, 198 p.

Henry Frances et Tator Carol (2009) Racism in the Canadian university: demanding social justice, inclusion, and equity, Toronto, University of Toronto Press, $224 \mathrm{p}$.

Hochschild Jennifer L. and Cropper Porsha (2010) Immigration regimes and schooling regimesWhich countries promote successful immigrant incorporation?, Theory and Research in Education, 8 (1), pp. 21-61.

Hoelscher Michael, Hayward Geoff, Ertl Hubert and DunbarGoddet Harriet (2008) The transition from vocational education and training to higher education: a successful pathway?, Research Papers in Education, 23 (2), pp. 139-151.

Ichou Mathieu (2014) Who they were there: Immigrants' educational selectivity and their children's educational attainment, European Sociological Review, $30(6)$, pp. 750-765. 
Jenkins Stephen P., Micklewright John and Schnepf Sylke V. (2008) Social segregation in secondary schools: how does England compare with other countries?, Oxford Review of Education, 34 (1), pp. 21-37.

Kamanzi Pierre Canisius, Bastien Nicolas, Doray Pierre et Magnan Marie-Odile (2016) Immigration et cheminements scolaires aux études supérieures au Canada : qui y va et quand? Une analyse à partir du modèle de risque proportionnel de Cox, Canadian Journal of Higher Education, 46 (2), pp. 209-232.

Kamanzi Pierre Canisius, Guégnard Christine, Imdorf Christian, Koomen Marteen et Murdoch Jake (2014) Démocratisation de l'enseignement supérieur et emprise des parcours: comparaison entre la France, la Suisse et le Canada, Télescope, 20 (2), pp. 170-188.

Kamanzi Pierre Canisius et Murdoch Jake (2011) L'accès à un diplôme universitaire chez les immigrants, in Kanouté Fassal et Lafortune Gina Éds., Familles québécoises d'origine immigrante: les dynamiques de l'établissement, Montréal, Presses de l'Université de Montréal, pp. 145-158.

Kamanzi Pierre Canisius, Pilote Annie, Uzenat Morgane et Gris Sandrine (2017) La démocratisation des études supérieures à l'aune de la différenciation et l'individualisation des parcours scolaires au Québec, Orientation scolaire et professionnelle, 46 (4), pp. 571-594.

King Karen M. and Newbold Bruce K. (2007) Internal migration dynamics of Canadian immigrant gateway: Toronto as an origin, way-station and destination between 1991 and 2001, Canadian Journal of Regional Sciences/Revue canadienne des sciences régionales, 30 (2), pp. 243-262.

Koopmans Ruud (2010) Trade-Offs between Equality and Difference: Immigrant Integration, Multiculturalism and the Welfare State in Cross-National Perspective, Journal of Ethnic and Migration Studies, 36 (1), pp. 1-26.

Lafortune Gina (2012) Rapport à l'école et aux savoirs scolaires de jeunes d'origine haïtienne en contexte scolaire défavorisé à Montréal, Thèse de doctorat inédite, Montréal, Université de Montréal.

Levels Mark, Dronkers Jaap and Kraaykamp Gerbert (2008) Immigrant children's educational achievement in western countries: origin, destination, and community effects on mathematical performance, American Sociological Review, 73, pp. 835-853.

Li Peter S. (2003) Deconstructing Canada's discourse of immigrant integration, Journal of International Migration and Integration, 4 (3), pp. 315-333.

Lowell Lindsay B. (2005) Policies and regulations for managing skilled international migration for work, New York, United Nations, Population Division Department of Economic and Social Affairs United Nations Secretariat, 24 p.

Lucas Samuel L. (2001) Effectively maintained inequality: education transitions, track mobility, and social background effects, American Journal of Sociology, 106 (6), pp. 1642-1690.

Lynch Kathleen and O'riordan Claire (1998) Inequality in higher education: A study of class barriers, British Journal of Sociology of Education, 19 (4), pp. 445-478.

Maclean Rupert and Pavlova Margarita (2013) Vocationalization of secondary and higher education: pathways to the world of work, in UNESCO-UNEVOC International Centre for Technical and Vocational Education and Training Eds., Revisiting global trends in TVET: Reflections on theory and practice, Bonn, UNESCO-UNEVOC, pp. 40-84. 
Martel Laurent et D'Aoust Carol (2016) L'immigration permanente et temporaire au Canada de 2012 à 2014 : Rapport de l'état de la population du Canada, Catalogue 91-209-X, Statistique Canada, 14 p., [en ligne]. URL : https://www150. statcan.gc.ca/n1/fr/catalogue/91-209-X201600114615

Mc Andrew Marie, Balde Alhassane, Bakhshaei Mahsa, Tardif-Grenier Kristel, Audet Geneviève, Armand Françoise, Guyon Sylvie, Ledent Jacques, Lemieux Georges, Potvin Maryse, Rahm Jrène, Vatz-Laaroussi Michèle, Carpentier Alain, et Rousseau Cécile (2015) La réussite éducative des élèves issus de l'immigration : dix ans de recherches et d'intervention, Montréal, Presses de l'Université de Montréal, $365 \mathrm{p}$.

Mc Andrew Marie, Garnett Bruce, Ledent Jacques et Ungerleider Charles (2008) La réussite scolaire des élèves issus de l'immigration : une question de classe sociale, de langue ou de culture?, Éducation et culture, 36 (1), pp. 177-196.

Mc Andrew Marie, Ledent Jacques, Murdoch Jake et Ait-Saïd Rachid (2011) La réussite scolaire des élèves issus de l'immigration au secondaire, Rapport final, Montréal, Ministère de l'Éducation, du Loisir et du Sport du Québec, 141 p.

Mc Andrew Marie, Ledent Jacques, Murdoch Jake, Ait-Saïd Rachid et Alhassane Balde (2013) Le profil et le cheminement scolaire des jeunes Québécois issus de l'immigration au secondaire : Un portrait statistique, Cahiers québécois de démographie, 42 (1), pp. 31-55.

Ministère de l'Immigration, de la Diversité et de I'Inclusion (MIDI) (2015). Portraits statistiques. L'immigration permanente au Québec selon les catégories d'immigration et quelques composantes 2010-2014, Québec, Gouvernement du Québec.

Narada Luckanachai and Rieger Matthias (2010) Making Migration a Development Factor: The Case of North and West Africa. A Review of International Policies, A Working Paper, Geneva, Programme for the Study of Global Migration, Graduate Institute of International and Development Studies, 52 p.

OCDE (2006) Where immigrant students succeed. A comparative review of performance and engagement in PISA 2003, Paris, OECD.

OCDE (2017) Perspectives de migrations internationales 2017, Paris, OCDE, 384 p.

OCDE (2015) Indicators of Immigrant Integration 2015, Paris, OECD, [online] last checked on 31/082017. URL: http://www.oecd.org/els/mig/lndicators-ofImmigrant-Integration-2015.pdf

Picard France, Trottier Claude et Doray Pierre (2011) Conceptualiser les parcours scolaires à I'enseignement supérieur, L'orientation scolaire et professionnelle, 40 (3), pp. 1-19.

Picot Garnett (2012) Immigrant status and secondary school performance as determinants of post-secondary participation: A Comparison of Canada and Switzerland, OECD Education Working Papers 77, OECD Publishing, 49 p., [online]. URL: http://dx.doi.org/10.1787/5k9909jhz4wl-en

Potvin Maryse, Magnan Marie-Odile et Larochelle-Audet Julie (2016) La diversité ethnoculturelle, religieuse et linguistique en éducation. Théorie et Pratique, Montréal, Fidès Éducation, 306 p.

Powell Justin J. W., Coutrot Laurence, Graf Lukas, Bernhard Nadine, Kieffer Annick and Solga Heike (2009) Comparing the relationship between vocational and higher education in Germany and France, Discussion Papers, Berlin, Wissenschaftszentrum Berlin für Sozialforschung [online]. URL: https://nbn-resolving.org/urn:nbn:de:0168-ssoar-259138 
Prost Antoine (1992) L'enseignement s'est-il démocratisé? Les élèves des lycées et collèges de l'agglomération d'Orléans de 1945 à 1990, Paris, Presses universitaires de France, $227 \mathrm{p}$.

Reitz Jeffrey G. (2001), Immigrant success in the economy knowledge economy: institutional change and the immigrant experience in Canada, 1970-1995, Journal of Social Issues, 57 (3), pp. 579-613.

Rothon Catherine, Heat Antony et Lessard-Philips Laurence (2009) The educational attainments of the "second generation": A Comparative study of Britain, Canada, and the United States, Teachers College Record, 111 (6), pp. 1404-1443.

Schneeweis Nicole (2013) Immigrant concentration in schools: Consequences for native and migrant students, Discussion paper 7230, Bonn, University of Linz, NRN Labor \& Welfare State and IZA, 42 p.

Schnell Philipp and Azzolini Davide (2015) The academic achievements of immigrant youths in new destination countries: Evidence from southern Europe. Migration studies, Migration Studies, 3 (2), pp. 217-240.

Schnepf Sylke (2007) Immigrants' educational disadvantage: an examination across ten countries and three surveys, Journal of Population Economics, 20 (3), pp. 527-545.

Shakya Yogendra B., Guruge Sepali, Hynie Michaela, Akbari Arzo, Malik Mohamed, Htoo Sheila, Azza Khogali, Mona Stella Abiyo, Murtaza Rabea and Alley Sarah (2010) Aspirations for higher education among newcomer refugee youth in Toronto: Expectations, challenges, and strategies, Refugee, 27 (2), pp. 65-78.

Simich Laura, Beiser Morton, Stewart Miriam and Mwakarimba Edward (2005) Providing social support for immigration and refugees in Canada: Challenges and directions, Journal of Immigrant Health, 7 (4), pp. 259-268.

Söhn Janina and Özcan Veysel (2006) The Educational Attainment of Turkish, Turish Studies, 7 (1), pp. 101-124.

Statistique Canada (2017a) Profil du recensement, Recensement de 2016, Statistique Canada, Ottawa, Statistique Canada, [en ligne]. URL : http://www12. statcan.gc.ca/census-recensement/2016/dp-pd/prof/index.cfm?Lang=F

Statistique Canada (2017b) Recensement de la population en 2016 : immigration et diversité ethnoculturelle, Ottawa, Statistique Canada, [en ligne]. URL : https:// www12.statcan.gc.ca/census-recensement/2016/ref/98-501/98-501-x2016008-fra. pdf

Thiessen Victor (2009) The pursuit of postsecondary education: A comparison of First Nations, African, Asian, and European Canadian Youth, Canadian Review of Sociology, 46 (1), pp. 5-37.

Zlotnik Honia (1995) The South-to-North Migration of Women, International Migration Review, 29, pp. 229-254.

Zlotnik Honia (1991) Trends in South to North Migration: The Perspective from the North, International Migration, 29 (2), pp. 317-331. 


\title{
Pierre Canisius Kamanzi, Marie-Odile Magnan, Annie Pilote et
} Pierre Doray

\author{
Immigration et morphologie des parcours scolaires \\ dans l'enseignement supérieur au Canada : \\ le cas de la province de Québec
}

Cet article vise à décrire et comprendre la morphologie des parcours des jeunes Québécois issus des familles immigrantes dans l'enseignement supérieur, ainsi que les facteurs qui contribuent à la structuration de ces parcours. Les résultats obtenus à partir des données du ministère de l'Éducation $(N=20387$ ) révèlent que ces jeunes poursuivent les études supérieures par la voie des parcours linéaires dans une proportion plus élevée que leurs pairs dont les parents sont nés au Canada. Toutefois, les différences sont nettement plus élevées à l'université qu'au collège d'enseignement général et professionnel (CEGEP). De plus, il existe des différences significatives parmi les jeunes d'origine immigrante selon I'origine géographique des parents. En revanche, il n'y a presque pas de différence à propos des parcours non linéaires. L'article suggère quelques pistes d'interprétation.

\section{Morphology of Immigrant Youth Pathways in Canadian Higher Education: The Case of the Province of Quebec}

This article aims to describe and understand the morphology of immigrant youth's pathways in higher education in Quebec, as well as the factors contributing to structuring them. The results obtained from the analysis of the Ministry of Education data ( $N=20,387$ ) show that these young people pursue higher education through linear pathways in larger proportions than their peers whose parents are born in Canada. However, the differences are much higher at the university than in General and Vocational College (known by their French acronym CEGEP: Collège d'enseignement général et professionnel). In addition, there are significant differences within immigrant youth according to the geographic origin of their parents. In contrast, there is almost no difference in nonlinear pathways. The article suggests some possible interpretations.

\section{Inmigración y morfología de las trayectorias académicas en la educación superior en Canadá: el caso de la provincia de Quebec}

Este artículo tiene por objeto la descripción y comprensión de la morfología de trayectorias de jóvenes de origen inmigrante en la educación superior, así que los factores que contribuyen a su estructuración. Los resultados recogidos a partir de datos del ministerio de Educación ( $N=20387$ ) demuestran que estos jóvenes cursan estudios superiores siguiendo trayectorias lineales en mayor proporción que sus pares cuyos padres nacieron en Canadá. Sin embargo, las diferencias son considerablemente más altas en la universidad que en los colegios de enseñanza general y profesional (CEGEP en Quebec). Además, se observan diferencias significativas entre los jóvenes de origen inmigrante según el origen geográfico de los padres. En cambio, no se observan diferencias en lo que concierne las trayectorias no lineales. Este artículo propone pistas de interpretación de dichos resultados. 